\title{
CONTROL SIMULTÁNEO CON INTELIGENCIA ARTIFICIAL PARA MITIGAR IRREGULARIDADES EN PROGRAMA VASO DE LECHE DE LOS GOBIERNOS LOCALES
}

\section{SIMULTANEOUS CONTROL WITH ARTIFICIAL INTELLIGENCE TO MITIGATE IRREGULARITIES IN THE VASO DE LECHE PROGRAM OF THE LOCAL GO- VERNMENTS}

\author{
José Gabriel Valladares Zúñiga \\ Universidad Nacional Mayor de San Marcos \\ Maestrista en auditoria con mención en auditoría en la gestión y control gubernamental
}

[Recibido: 10/09/2017 Aceptado:20/04/2018]

\section{RESUMEN}

El artículo tiene como objetivo describir cómo el control simultáneo con inteligencia artificial en el Programa del Vaso de Leche, mitigará las irregularidades halladas en los operativos de control posterior a las exoneraciones de los procesos de selección en los cuales se comprobó que afectó el abastecimiento oportuno de los alimentos de dicho Programa. Esas irregularidades existen porque los funcionarios de las municipalidades no son conscientes que el Vaso de Leche es el desayuno de los infantes que viven en extrema pobreza y no son supervisados oportunamente por los Órganos del Sistema Nacional de Control y ven en este Programa la oportunidad para beneficiarse previo acuerdo con los proveedores. Ante ello, este artículo propone procesos por medio de inteligencia artificial en cuya Base de Datos de Conocimiento se alojarán las estructuras de las normas relativas al PVL. Los hechos que los funcionarios ediles tienen como actividad se ingresarán a la Base de Datos de Hechos. Esos datos serán validados por dicho sistema, mostrando la conclusión correcta o las alertas que les serán enviadas para que las corrija y concluya correctamente su actividad. Así, se cumplirán oportunamente las funciones, y la municipalidad llegará a abastecerse oportunamente, durante todos los meses del año.

Palabras clave : Control posterior, control simultáneo, planificación, programación, abastecimiento, sistemas expertos, inteligencia artificial.

\begin{abstract}
This article describes how the simultaneous control with artificial intelligence in the "Vaso de leche" (glass of milk) Program will mitigate the irregularities found in the control operations after the exonerations of the selection processes, it was found that this affected the supply timing of the foods of the Program. These irregularities exist because the administrators of the municipalities are not aware that the "Vaso de leche" is the breakfast of the infants who live in extreme poverty and are not supervised in an appropriate timing management by the Bodies of the National Control System, and they see in this Program the opportunity to benefit, prior agreement with the suppliers. In view of this, this article proposes processes with artificial intelligence where Database Knowledge will host the norms' structures related to the PVL. The facts about municipal official's activities will be entered into the Fact Database. These data will be validated the system, showing the correct conclusion or the alerts that will be sent to them to correct and correctly conclude their activity. Thus, the functions will be fulfilled in a timely manner, and the municipality will arrive to supply itself opportunely, during all the months of the year.
\end{abstract}

Keywords: Post control, simultaneous control, planning, programming, supply, expert systems, artificial intelligence.

\section{INTRODUCCIÓN}

La Contraloria(2014) refiere que el control selectivo gubernamental posterior electrónico, vía rendiciones en formatos web, no mitiga las irregu- laridades administrativas cometidas por los funcionarios municipales desde la programación del abastecimiento -que se inicia el 2 de enero del año X- hasta la contratación de los alimentos del Programa Vaso de
Leche - PVL -que finaliza el 31 de diciembre de $\mathrm{X}+1$, como si lo mitigaría el control simultáneo (Contraloria, 2015) electrónico.

El PVL (Contraloría, 2009) es un Pro-

( L Los autores. Este artículo es publicado por la Revista Quipukamayoc, Universidad Nacional Mayor de San Marcos. Este es un artículo de acceso abierto, distribuido bajo los términos de la Licencia Creative Commons Atribución-NoComercial-Compartirlgual 4.0 Internacional.(http://creativecommons.org/licenses/by-nc-sa/4.0/), que permite el uso no comercial, distribución y reproducción en cualquier medio, siempre que la obra original sea debidamente citadas. 
grama de Asistencia Social en el Perú sin costo alguno para todos los beneficiarios (primera prioridad son los niños de 0 a 6 años de edad, madres gestantes y en período de lactancia) con derecho a la provisión diaria por parte del Estado, a través de los municipios, de 250 centímetros cúbicos de leche o alimento equivalente preparado; sin embargo, los funcionarios ediles inoportunamente recurren a pretextos fuera de la normativa (irregularidades) para exonerarse de realizar procesos de selección bajo la causal de "Desabastecimiento".Contraloría (2014)

Los informes de auditorías a las exoneraciones del 2003 al 2011 indican que las modalidades de irregularidades (hechos indebidos) alcanzó al valor referencial de S/57 888384 en Lima Metropolitana y fueron, entre otras, las siguientes:

a. Falta de planificación oportuna de las etapas de programación y actos preparatorios de los procesos de selección.

b. Se demoraron en aprobar el Presupuesto Institucional de Apertura.

c. Se demoraron en aprobar el Plan Anual de Adquisiciones y Contrataciones (PAAC).

d. Los Procesos de selección no fueron incluidos en el PAAC.

e. Se invocó la causal "Situación de urgencia”, y después del cambio de normativa, la de "Desabastecimiento", emitiendo exoneraciones de procesos de selección, a través de Acuerdos de Consejo Municipal sin reunir los requisitos previstos en la normativa.

El efecto negativo en los beneficiarios es la demora en la entrega del alimento y su baja calidad, lo que conlleva a una baja ingesta de macro y micronutrientes (proteínas, etc.) y por tanto un sub-desarrollo físico y mental del infante (ONU, 2001).

El presupuesto del PVL se mantiene todos los años desde el 2007 (MEF, 2007) y se da a conocer a los alcaldes, solo los incrementos, desde el mes de junio de todos los años por el Ministerio de Economía y Finanzas; para esto, los alcaldes y sus funcionarios, antes de junio (ósea en enero) ya debieron haber iniciado la Planificación (Burgwal, 1999) - programar las actividades del PVL y estar ejecutando las actuaciones previas al proceso de selección-; por lo que, tendrían tiempo para ejecutar dicho proceso (sin exonerarse de los mismos).

El Modelo Procedimental de control simultáneo electrónico es un software prototipo de Sistema Experto, rama de la inteligencia artificial Cuellar (2003), que se desarrollará enfocando las dos primeras fases del proceso del Programa Vaso de Leche: la Programación y las Actuaciones Preparatorias del Proceso de Selección para la selección de proveedores, porque allí se han presentado —desde el 2003 hasta el 2011 - las veinte (20) irregularidades administrativas más significativas.

El orden cronológico de las normas para lograr nuestro Objetivo Final debe ser codificado (estructurado) por el Experto Humano (Quintanar, 2007) utilizando las letras mayúsculas del alfabeto, en un encadenamiento hacia adelante Neira (2014), tomando solo los mandatos imperativos de cada norma regla (obligaciones de hacer), a fin que el Ingeniero del Conocimiento los ingrese en la Base de Datos de Conocimiento (BDC), de la siguiente manera:

La jerarquía de las normas (variables) no se toma en cuenta; puesto que las reglas formarán un encadenamiento cronológico (una agenda o una lista de las normas) para lograr nuestro Objetivo Final: "La Convocatoria oportuna del proceso de selección”.

En cada variable (norma legal: A, B, $\mathrm{C}$, etc.) hay muchas reglas (obligaciones o deberes) y la estrategia del motor de inferencia es el hacer un encadenamiento de reglas hacia adelante con una agenda o lista, dependiendo de la regla anterior; es decir la dependencia propuesta es la implícita hacia un objetivo, no dejando que la tarea irregular (hecho irregular) ingresada permanezca, puesto que generará validaciones y una alerta a través del Módulo o Motor de Explicación Díaz, (2010) que se mostrará al usuario por la interfaz del sistema, y solo la alerta paralizará al sistema.

Base de Datos de Conocimiento (BDC)

Estas Variables y sus Reglas son introducidas por el Ingeniero del Conocimiento (Ayala, 2006), en la Base de Datos de Conocimiento (BDC) del Sistema Experto, a fin que se tenga un parámetro con el cual se puedan comparar los Hechos irregulares.

\section{Base de Datos de Hechos (BDH)}

Teniendo llena y culminada la BDC, se desarrolla una herramienta de Aplicación (software Bizagui) que contenga a la Base de Datos de Hechos (BDH). Cuando los Hechos son ingresados y guardados en la Memoria de Trabajo (Padilla Díaz, 2010) por el usuario (funcionario municipal o OSB) en el documento pre establecido alojado en la $\mathrm{BDH}$, el Motor de Inferencia, hace una comparación con las reglas de cada variable alojada en la BDC. Aquí, la función del usuario del sistema experto del PVL es cumplir con las normas legales que están en la BDC. El Hecho (tarea) que realiza cada usuario del PVL en el sistema es una secuencia fija con tiempo prefijado, y su cumplimiento es controlado por las reglas encadenadas de la sub actividad: "Programación y Actuaciones Preparatorias del proceso de selección del PVL".

Para ello, se presenta en forma estructurada la proposición (o premisa) $\mathrm{Si}$ 1a (antecedente); entonces $1 \mathrm{~b}$ (consecuente) a todo lo subrayado se le conoce como Regla 1 de la Variable A (Regla 1 de A) en la BDC. El encadenamiento de reglas funciona de la Regla 1 a la Regla 2.

El Motor de Inferencia realiza el trabajo de buscar (o cotejar) su par, desde el hecho 1a de la Memoria de Tra- 
bajo de la $\mathrm{BDH}$ hacia los antecedentes o condiciones (Si) de cada Regla de la BDC, empezando desde la Regla 1 de A, y cuando se empareja la tarea 1a, con la regla la, automáticamente se habilita la regla siguiente $1 \mathrm{~b}$ (si 1a, entonces 1b). 1a es la "condición" y 1 b es la "conclusión" de la Regla 1 de A. Seguidamente se espera el ingreso de la siguiente tarea en la interfaz de usuario, la 1b, y cuando la guarda en la Memoria de Trabajo de la $\mathrm{BDH}$, esta se empareja con la regla $1 b$ de la Regla 2 de A y automáticamente se deshabilita la Regla 1 de A, lo que significa que cumplió correctamente con la regla: "haber recibido de las OSB las solicitudes de registro adjuntando los documentos electrónicos"; quedando a la espera de la siguiente tarea $2 \mathrm{a}$.

Hasta aquí, se dice que el Motor de Inferencia aplicó el método "Modus Ponendo Ponens" que significa "Método Afirmando Afirma” (Darkjrof, 2011). Ese cumplimiento correcto de la norma se codifica como Conclusión $1 \mathrm{~b}$, y viene a ser el primer objetivo cumplido (objetivo parcial) que se convierte en un Hecho conocido en la BDC al deshabilitarse la Regla 1, en todo el encadenamiento hacia adelante.
Si el Motor de Inferencia no encuentra su par en la BDC, esta queda como Conclusión Temporal y se añade esta Nueva Tarea (Nuevo Hecho) a la lista de condiciones (agenda de Hechos o Tareas) de la $\mathrm{BDH}$, lo que provoca que todo el sistema experto se paralice, para que posteriormente, el Experto Humano, evalué la secuencia y tiempo desde el Hecho 1a. De ser "razonable" este Nuevo Hecho, el Ingeniero del Conocimiento agregará la Nueva Regla a la BDC a fin que se empareje y desparalice el sistema.

La estrategia de búsqueda del Motor de Inferencia hacia el objetivo parcial (Conclusión 5b) no es desordenada (no aleatoria); sino, se estructura para que la búsqueda sea en orden desde los documentos pre-establecidos en donde se ingresan los hechos, hacia las reglas habilitadas encadenadas hacia adelante. Con Nuevos Hechos, el Motor de Inferencia produce o crea conocimiento y su próxima búsqueda la hará sólo en las condiciones de las reglas habilitadas (es decir, a excepción de la Regla 1 de A, ni de la Nueva Regla que ya quedaron marcadas o deshabilitadas en la BDC durante el período de tiempo anual del sistema experto del PVL).

Esta estrategia del Motor de Inferencia es denominada "impulsado por datos"; es decir, inicia con el primer hecho o dato, y una vez satisfecha la primera regla (ósea ocurrida la Conclusión), ésta ya no será buscada por el motor, pasando a buscar en las siguiente(s) Regla(s), porque las reglas están encadenadas para llegar a la Conclusión Final (Objetivo Final). Por ejemplo, las Conclusiones $1 \mathrm{~b}$ y $3 \mathrm{~b}$ se añaden como hechos "conocidos" a la BDC, porque fue satisfecha la Regla 1 de A y la Regla 5 de A respectivamente, cuando el trabajador municipal ingresó su tarea (Hecho $1 \mathrm{~b}$ y Hecho $3 \mathrm{~b})$, respectivamente.

Las Conclusiones temporales $2 \mathrm{~b}$ y $4 \mathrm{~b}$ no se añaden como hechos "conocidos" para la BDC, porque no fue satisfecha la Regla 3 de A y la Regla 7 de A, respectivamente; ello sucederá porque el trabajador municipal: no ingresa su tarea; la ingresa a destiempo; ingresa una Nueva Tarea (Nuevo Hecho) que no está en la agenda o lista; o ingresa mal su tarea (Hecho $2 \mathrm{~b}$ y Hecho 4b), respectivamente; siendo que estos casos generarán alertas a

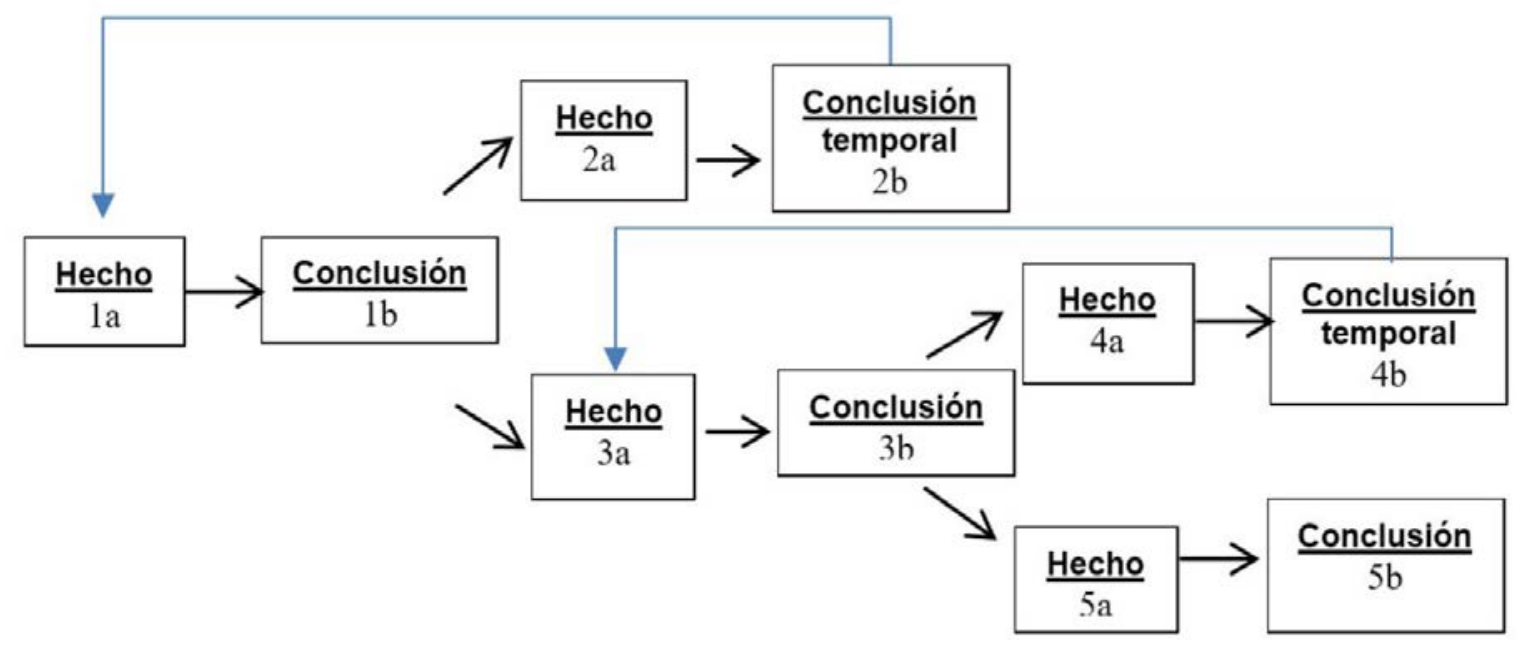

Figura 1. Del Motor de Explicación para los órganos de control. Fuente: Base de Datos de Hechos (BDH) - Elaboración propia. 


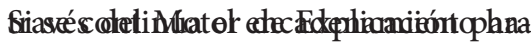

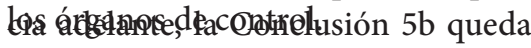
habilitada para recibir a la Tarea o Hecho de la siguiente Regla 10 de la Variable B (Regla 10 de B) en todo el encadenamiento hacia adelante, y así sucesivamente se llegará al Objetivo Final, que es: "La Convocatoria oportuna (hasta el último día hábil de setiembre) del proceso de selección".
Se diseñó el modelamiento de todas las Tareas (Hechos) secuenciales de la Programación del Abastecimiento del PVL, en el aplicativo Bizagi Modeler siguiente:

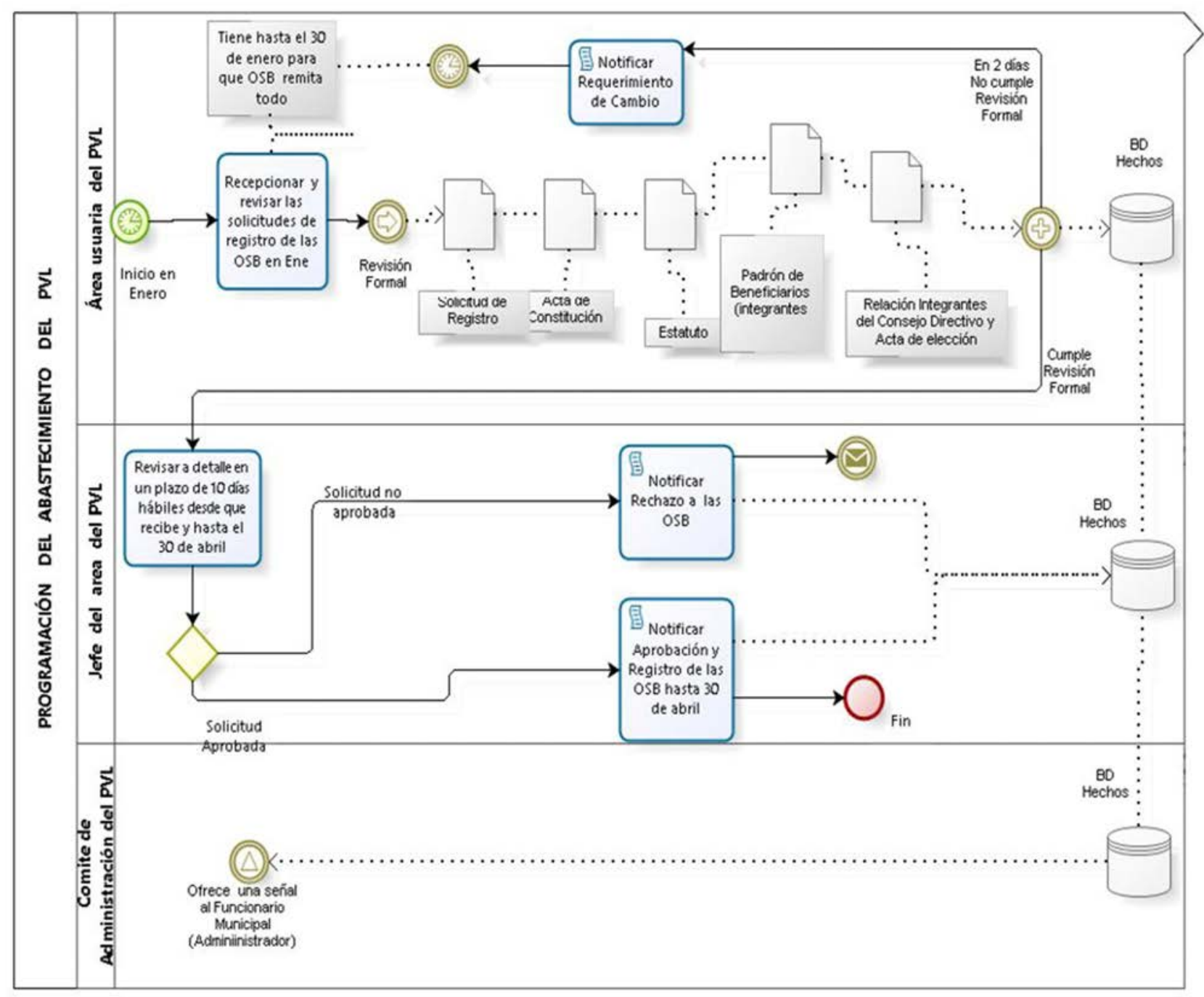

Figura 2. Programación del Abastecimiento del PVL.

Fuente: Elaboración propia.

La figura anterior muestra los documentos electrónicos pre-establecidos, con firmas certificadas (Torres Alvárez, 2005) y con tiempos prefijados, a fin que generada la alerta, el órgano de control acceda con su usuario y su contraseña, y monitoree en tiempo real, cualquiera de estos documentos electrónicos que se guardaron en la $\mathrm{BDH}$; por ejemplo, las hojas Word, Excel, Access, Base de Datos Web de Contactos, la Solicitud de Registro de OSB (o Solicitud de Inscripción de OSB), el Padrón de Beneficiarios, los
Estatutos, etc. Se diseñó el modelamiento de todas las Tareas (Hechos) secuenciales de las Actuaciones Preparatorias para el Proceso de Selección del PVL, tal como sigue: 


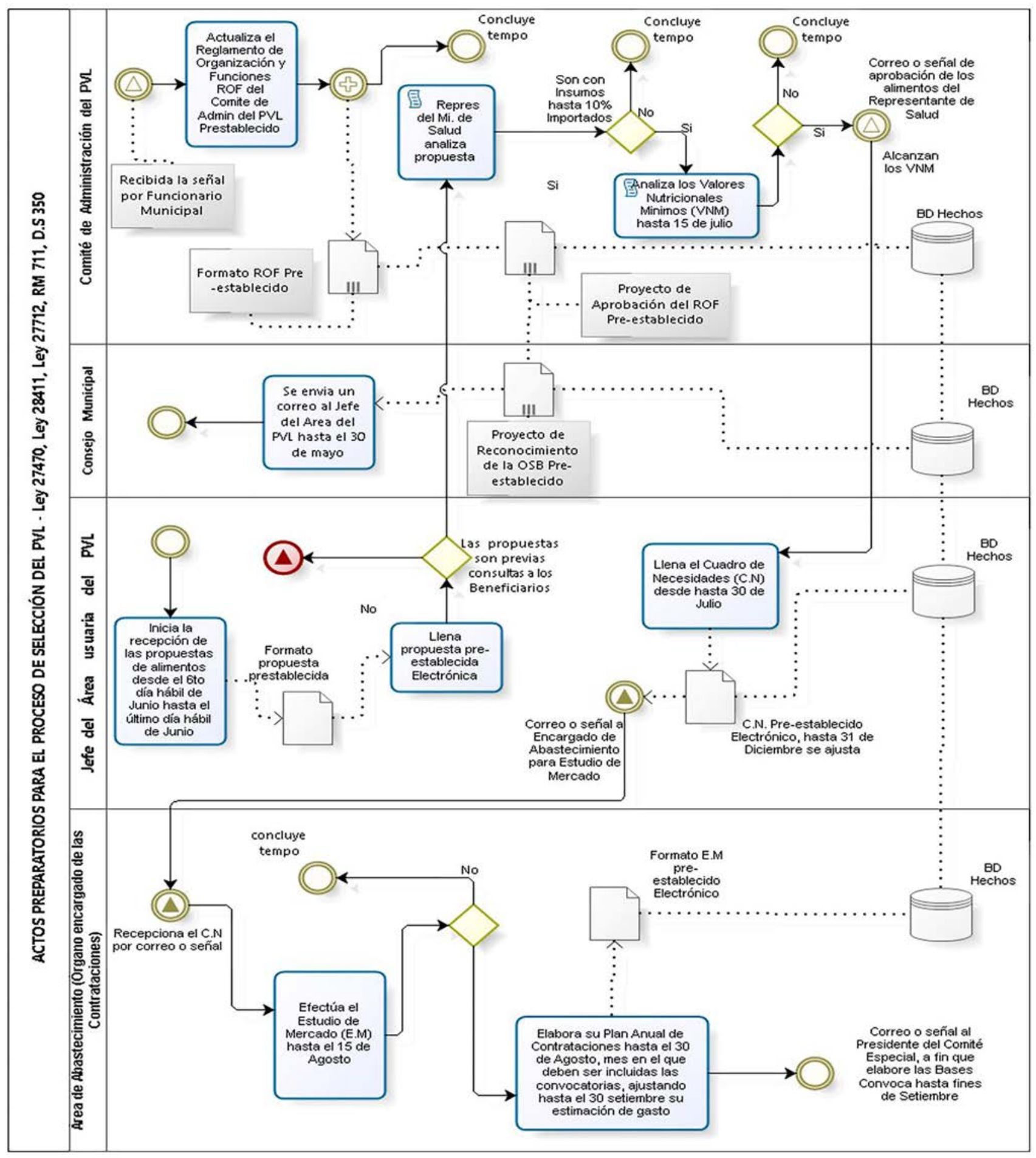

Figura 3. Actuaciones preparatorias.

Fuente: Elaboración propia. 
El Formato ROF tiene firma electrónica como evidencia Valencia (2012) y cuando el órgano de control ingrese al sistema con un usuario y contraseña, se abrirá y podrá monitorear o controlar la tarea que está realizando el trabajador municipal y su regla encadenada. Podrá observar si hay Conclusiones Temporales y sus alertas a través del Motor de Explicación.

La interfaz del usuario a través del Motor de Explicación mostrará los mensajes de validación en los terminales de computadoras de las municipalidades, y solo admitirá el ingreso de datos inválidos hasta dos (2) veces. $\mathrm{Al}$ tercer ingreso inválido, se genera- rá la Conclusión Temporal y su alerta Torres, Montes, y Díaz (2012) de irregularidad, que solo será mostrado a los Órganos de Control. La estructura estratégica de sistema experto propuesta es la siguiente:. (Rojas 2012)
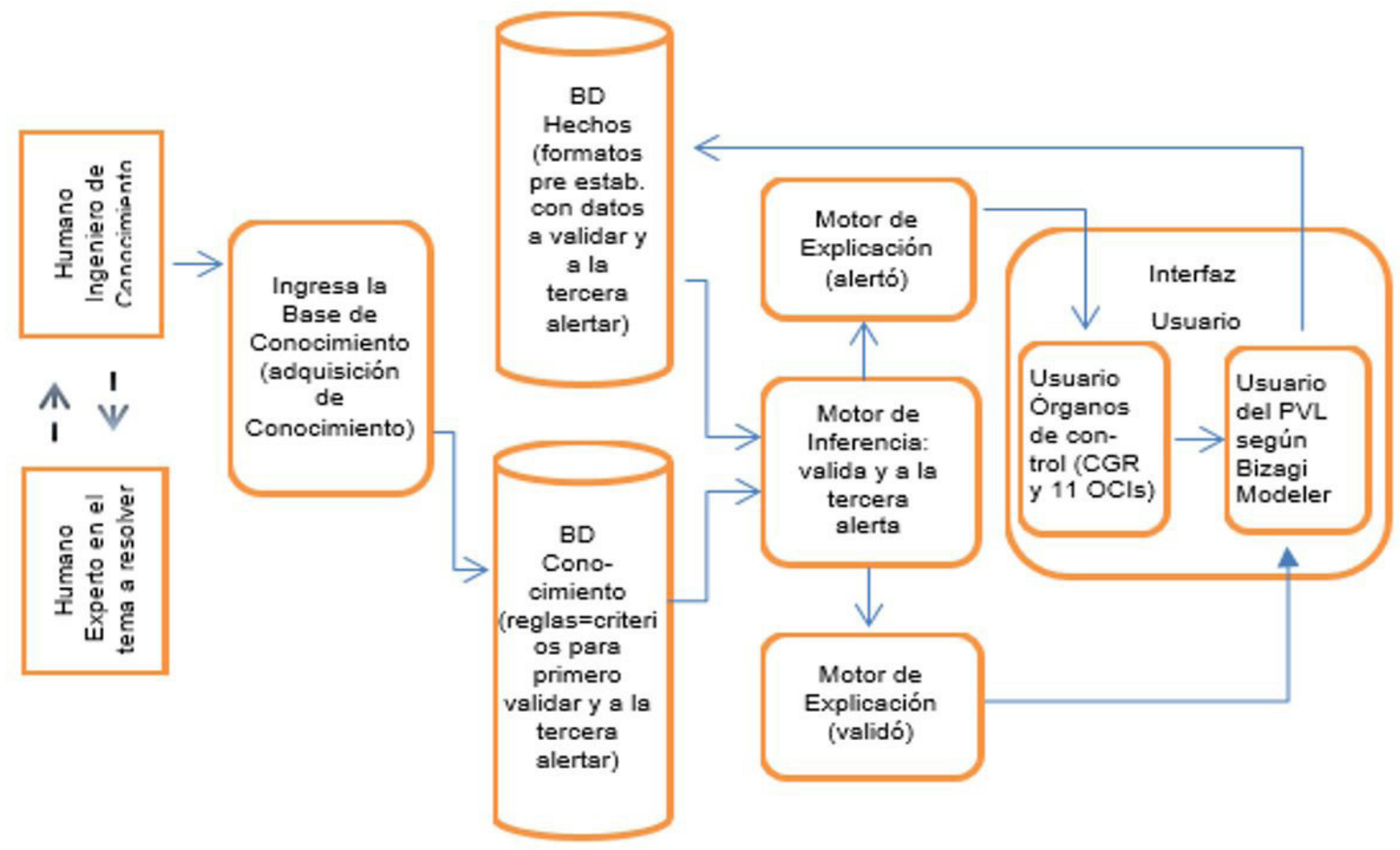

Figura 4. Sistema Experto.

Fuente: Elaboración propia.

\section{MATERIAL Y MÉTODOS}

Se utilizó como material formatos de encuestas y el método de investigación partiendo de su objetivo fue el "No Experimental" porque no manipula intencionalmente ninguna de las tres (3) variables, ni la primera, que es el control gubernamental posterior, ni la segunda que son las irregulari- dades, ni la tercera, que es el control simultaneo que se "propone". Para el objetivo específico n. ${ }^{\circ} 1$, el diseño es el "Longitudinal de Tendencia", el nivel de estudio inicial fue el "Descriptivo" y finalizó con el "Correlacional” (Tiempo versus Irregularidades) del 2003 al 2016. Para el objetivo específico n. ${ }^{\circ} 2$, el diseño es el "Transversal (o Transeccional)"; el nivel de estudio se inicia como "Exploratorio" porque busca una estrategia del sistema experto, no existente; pasa por el "Descriptivo” al describir el ¿cómo? dicha estrategia mitigará las irregularidades con dicho sistema; y finaliza con un "Correlacional" entre sus indicadores (Rigurosidad versus Continuidad). 


\section{RESULTADOS}

Tenemos como Hipótesis :

$($ HEA0) $=$ alterna nula: CANT_IRREGULcontrolsimultaneo $\geq$ CANT_IRREGUL_controlposterior $($ HEA1) $=$ alterna de trabajo: CANT_IRREGULcontrolsimultaneo $<$ CANT_IRREGUL_controlposterior

Tabla 1. Cantidad de irregularidades 2003-2011.

Fuente: Elaboración propia.

\begin{tabular}{lccccccc} 
AÑOS TRANSCURRIDOS & $2003-2004$ & $2005-2006$ & 2007 & 2008 & 2009 & 2010 & 2011 \\
$\begin{array}{l}\text { Número de acciones control } \\
\text { posterior }\end{array}$ & 1 & 1 & 1 & 1 & 1 & 1 & 1 \\
\cline { 2 - 8 } Cantidad de irregularidades & 14 & 8 & 46 & 31 & 19 & 6 & 6 \\
\hline
\end{tabular}

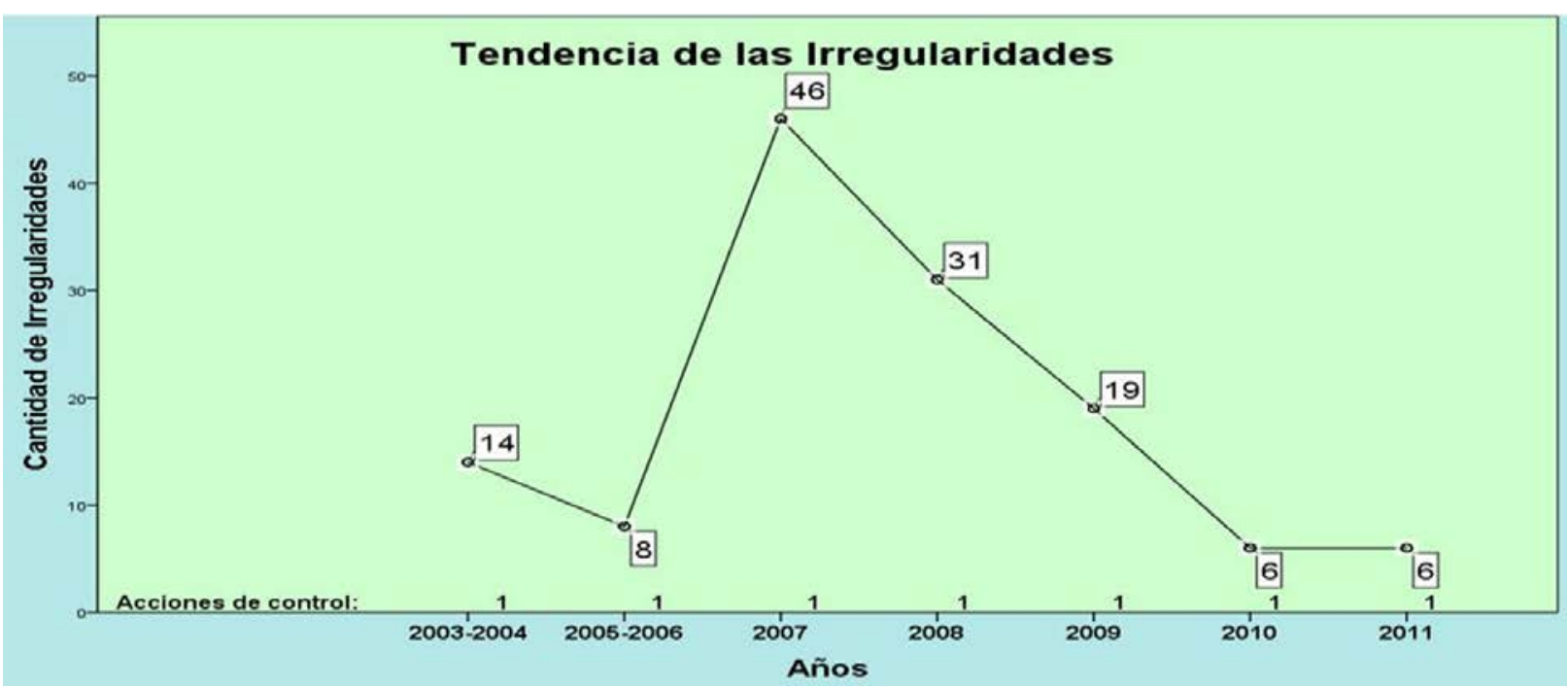

Figura 5. Tendencia de las irregularidades.

Fuente: Irregularidades de los Informes de la CGR, generado de la herramienta SPSS - Elaboración propia.

La cantidad de irregularidades de 46 fue excesiva en las 33 Municipalidades que conforman la población en estudio de Lima Metropolitana. Para el año 2018 y siguientes la tendencia es de 6 irregularidades si seguimos aplicando control posterior vía rendición de cuentas en un sistema web electrónico convencional.

Sin embargo, vemos que las encuestas exploratorias en el 2016 a la muestra de 11 Jefes de Órgano de Control Institucional (OCI), arrojaron los siguientes resultados:

Tabla 2. Encuestas exploratorias en el 2016 -muestra de 11 Jefes de Órgano de Control Institucional (OCI). Fuente: Elaboración propia.

\begin{tabular}{|c|c|c|c|c|c|c|c|c|c|}
\hline & & \multicolumn{3}{|c|}{$\begin{array}{l}\text { Continuidad al día (en horas) } \\
\text { que se espera ejecutar el } \\
\text { Sistema experto }\end{array}$} & \multicolumn{3}{|c|}{$\begin{array}{l}\text { Rigurosidad del diseño del } \\
\text { Modelo Procedimental del } \\
\text { Sistema experto }\end{array}$} & \multicolumn{2}{|c|}{$\begin{array}{l}\text { Cantidad de Irregularidades Pre- } \\
\text { dichas con el Sistema experto }\end{array}$} \\
\hline & & $8 \mathrm{~h}$ & $4 \mathrm{~h}$ & $2 \mathrm{~h}$ & Baja & Media & Alta & De 6 a más De 3 a 5 & De 0 a 2 \\
\hline 1 & M.D. DE ATE-VITARTE & 1 & & & & & 1 & & 1 \\
\hline 2 & M.D. DE BARRANCO & & & 1 & & 1 & & & 1 \\
\hline 3 & M.D. DE INDEPENDENCIA & & 1 & & & 1 & & & 1 \\
\hline
\end{tabular}




\begin{tabular}{|c|c|c|c|c|c|c|c|c|c|c|}
\hline 4 & M.D. DE LA MOLINA & & & 1 & & & 1 & & & 1 \\
\hline 5 & M.D. DE LA VICTORIA & & & 1 & & & 1 & & & 1 \\
\hline 6 & M.D. DE LINCE & & & 1 & & & 1 & & & 1 \\
\hline 7 & M.D. DE MAGDALENA & & & 1 & & & 1 & & 1 & \\
\hline 8 & M.D. DE MIRAFLORES & & & 1 & & & 1 & & 1 & \\
\hline 9 & M.D. DE PUEBLO LIBRE & & & 1 & & & 1 & & & 1 \\
\hline 10 & M.D. DE SURQUILLO & & & 1 & & & 1 & & & 1 \\
\hline \multirow[t]{2}{*}{11} & M.D. DE SURCO & & & 1 & & & 1 & & & 1 \\
\hline & Total conteo & 1 & 1 & 9 & 0 & 2 & 9 & 0 & 2 & 9 \\
\hline
\end{tabular}

La cantidad de irregularidades de 46 fue excesiva en las 33 Municipalidades que conforman la población en estudio de Lima Metropolitana. Para el año 2018 y siguientes la tendencia es de 6 irregularidades si seguimos aplicando control posterior vía rendición de cuentas en un sistema web electrónico convencional.

Sin embargo, vemos que las encuestas exploratorias en el 2016 a la mues- tra de 11 Jefes de Órgano de Control Institucional (OCI), arrojaron los siguientes resultados:

Tabla 3. Estadísticas de muestras emparejadas.

Fuente: Exploratorio.sav - Elaboración propia.

\begin{tabular}{clccccc} 
& & Media & N & Desviación estándar & Media de error estándar \\
\cline { 3 - 7 } Par 1 & CANT_IRREGUL_controlposterior & 5,45 & 11 & 1,214 &, 366 \\
\cline { 2 - 7 } & CANT_IRREGULcontrolsimultaneo & 2,18 & 11 &, 405 &, 122 \\
\hline
\end{tabular}

Tabla 4. Correlaciones de muestras emparejadas.

Fuente: Exploratorio.sav - Elaboración propia.

\begin{tabular}{ccccc}
\multirow{2}{*}{ Par 1} & CANT_IRREGUL_controlposterior & N & Correlación & Sig. \\
\cline { 2 - 5 } & CANT_IRREGULcontrolsimultaneo & 11 & $-1,000$ &, 000 \\
\hline
\end{tabular}

Tabla 5. Prueba de muestras emparejadas.

Fuente: Exploratorio.sav - Elaboración propia.

\begin{tabular}{|c|c|c|c|c|c|c|c|c|c|}
\hline & \multicolumn{5}{|c|}{ Diferencias emparejadas } & \multirow[t]{3}{*}{$\mathrm{t}$} & \multirow[t]{3}{*}{$\mathrm{gl}$} & \multirow[t]{3}{*}{$\begin{array}{l}\text { Sig. (bila-teral) } \\
=\text { P-VALOR }\end{array}$} \\
\hline & & \multirow[t]{2}{*}{ Media } & \multirow[t]{2}{*}{$\begin{array}{c}\text { Desviación } \\
\text { estándar }\end{array}$} & \multirow{2}{*}{$\begin{array}{l}\text { Media } \\
\text { de error } \\
\text { estándar }\end{array}$} & \multicolumn{2}{|c|}{$\begin{array}{c}95 \% \text { de intervalo de } \\
\text { confianza de la } \\
\text { diferencia }\end{array}$} & & & \\
\hline & & & & & Inferior & Superior & & & \\
\hline Par 1 & $\begin{array}{l}\text { CANT_IRREGUL_controlposterior } \\
\text { vs } \\
\text { CANT_IRREGULcontrolsimultaneo }\end{array}$ & 3,273 & 1,618 & , 488 & 2,186 & 4,360 & 6,708 & 10 &, 000 \\
\hline
\end{tabular}


Dado que el p valor es menor al alfa, existe evidencia estadística para aceptar HEA1, es decir, la cantidad promedio de irregularidades con control simultáneo es siempre menor a la cantidad promedio de irregularidades con control posterior en 3,273 .

Tabla 6. Cantidad de Irregularidades.

Fuente: Exploratorio.sav - Elaboración propia.

\begin{tabular}{cccccc} 
& & Frecuencia & Porcentaje & Porcentaje válido & Porcentaje acumulado \\
& \multirow{3}{*}{ Válido } & 2 & 18,2 & 18,2 & 18,2 \\
\cline { 2 - 6 } & De 3 a 5 inclusive & 9 & 81,8 & 81,8 & 100,0 \\
\cline { 2 - 6 } & Dotal 2 inclusive & 11 & 100,0 & 100,0 & \\
\hline
\end{tabular}

Se aprecia que dos Jefe de OCI respondieron que la cantidad de irregularidades que el sistema experto alertará a la CGR, es 3 a 5 irregularidades; pero nueve Jefes de OCI indicaron que por su Rigurosidad de su diseño, solo quedarán 0 a 2 irregularidades. El nivel de significancia " $\alpha$ " es de 0,15 $(0.15 \%)$ y para este nivel, nuestro " $p$ " valor resultó 0,000 (menor); por lo tanto, existe evidencia estadística para aceptar nuestra Hipótesis Especifica Alterna HEA1, de que "con control simultáneo, se mitigará las irregularidades, que si se aplicase control posterior".

\section{DISCUSIÓN}

No hay duda, con el control simultáneo con sistema experto de inteligencia artificial, llegaremos a mitigar irregularidades e incluso podría evitarlas, en aras de lograr un abastecimiento y consumo oportuno de los alimentos del Vaso de Leche.

Se mitigan las irregularidades creando un nuevo proceso y cambiando la normativa del Decreto Supremo n. 056-2017-EF vigente, el cual menciona en su artículo 5 que debe efectuarse las convocatorias a procesos de selección el año fiscal siguiente, al año en el cual, las áreas usuarias de las Entidades programaron en el Cuadro de Necesidades sus requerimientos de raciones de dichos alimentos. Este sistema experto de inteligencia artificial se desarrollará considerando el proceso de que Cuadro de Necesidades, convocatoria del proceso de selección y firma del Contrato, sean hechos, actos o tareas, ingresadas en un solo año calendario.

De lo contrario, seguiremos con este Decreto en contraposición al Principio de "Anualidad" que regula el Sistema Nacional de Presupuesto, en el que, correctamente se norma afectar todos los gastos generados con cargo al presupuesto autorizado de la municipalidad (PIA), considerando que la afectación es el "Compromiso" (contrato) que se firma antes del $31 \mathrm{de}$ diciembre del año X; y el "Cuadro de Necesidades" muestra requerimientos de los alimentos del PVL que se recaban desde el 2 de enero del año X (mismo año).

El hecho que se tenga que convocar a un proceso de selección después que se apruebe el PIA, y que el PAC se apruebe después de los 15 días hábiles siguientes a la aprobación del PIA $(\mathrm{X}+1)$, está en oposición al principio de "Predictibilidad del gasto público" que señala: la "Programación de Compromisos Anual (PCA) permite a los pliegos tener la certidumbre sobre el límite anual para realizar compromisos anualizados (para firmar contratos de abastecimiento); ello está respaldado con la "Opinión n. 047-2016/DTN” del OSCE. Solo así se mitigarán las irregularidades que se producen cuando se exoneran del proceso de selección por causal de desabastecimiento.

\section{REFERENCIAS BIBLIOGRÁFICAS}

Burgwal, G. (1999). Planificación Estratégica y Operativa aplicada a los Gobiernos Locale. Quito: Ediciones Abya Yala.

Contraloría. (2014). "Exposición de Motivos del Proyecto de Ley $n .^{\circ}$ 4361/2014-CGR", "Ley que faculta a la Contraloría General de la República para disponer la intervención de las entidades por el control gubernamental mediante el ejercicio del control previo y simultáneo". Recuperado el 2015, de Proyecto de Ley n. ${ }^{\circ}$ 4361/2014-CGR: http://www. congreso.gob.pe/proyectosdeley

Contraloría. (2014). Resolución de Contraloría n. ${ }^{\circ}$ 273-2014-CG. Normas Legales del "El Peruano".

MEF. (2007). DU019_2007.pdf. Obtenido de https://www.mef.gob.pe/ es/por-instrumento/decreto-de-urgencia/1385-d-u-n-019-2007/file

ONU. (2001). Guía para la Gestión 
Municipal de Programas de Seguridad Alimentaria y Nutrición”, Capitulo 5 "Manejo De Programas de Asistencia Alimentaria a Nivel Municipal , Santiago de Chile::Cecilo Morón .

Padilla, A. (2010). Sistema Experto para la Interpretación Mamográfica. México D.F., México, México.

Peña, A. (2006). “Sistemas basados en Conocimiento - Una Base para su Concepción y Desarrollo". México D.F: Talleres Gráficos de la
Dirección de Publicaciones del Instituto Politécnico Nacional Revillagigedo 83.

Quintanar, T. (2007). Sistemas Expertos y sus Aplicaciones. Pachuca de Soto, México D.F:Hidalgo.

Rojas, J. (2012). Sistema experto para el control de los procesos de monitoreo, control y evaluación de desempeño de los Órganos de Control Institucional del Perú. Lima.

Torres, H. (2005). El Sistema de
Seguridad Jurídica en el Comercio Electrónico". Lima, Fondo Editorial de la Pontificia Universidad Católica del Perú, Primera Edición,Lima

Torres, R. G., Montes, S. G., \& Díaz, P. R. (2012-2013). Sistema Concurrente de Detección de Instrusiones con Correlación de Alertas en Entornos Distribuidos. Madrid: Madrid . 